sICAM-1, IFN- $\gamma$, IL-10, and TGF- $\beta 1$ in all three groups ( $\mathrm{p}$ all $<0.05)$. It was found that CTLA-4Ig could decrease the serum concentration of CRP, sICAM-1 and IFN- $\gamma$ and increase IL-10 and TGF- $\beta 1$, but IgG1 and PBS.

Conclusions CTLA-4Ig fusion protein could inhibit the (del) atherosclerosis progression in apoE(-/-) mice fed on cholesterol diet and it's effect might be associated with blocking B7/CD28, antiinflammation, promoting Th2 polarisation and affecting regulate $\mathrm{T}$ cells.

\section{e0187 ENHANCED EXTERNAL COUNTERPULSATION PROTECTS VASCULAR ENDOTHELIAL CELLS FROM APOPTOSIS IN HYPERCHOLESTEROLEMIC PIGS}

doi:10.1136/hrt.2010.208967.187

Yan Xiong, Yan Xiong. The First Affiliated Hospital of Sun Yatsen University, Guangzhou, China

Objective Evidences have proved that Enhanced external counterpulsation (EECP) improves endothelial dysfunction and repairs intimal damage by increasing vascular endothelial shear stress. Based on the assumption that unbalanced apoptosis of vascular endothelial cells (VECs) may have played a pivotal role in the pathogenesis of atherosclerotic lesions, we hypothesised that longterm EECP protects VECs from apoptosis in hypercholesteronlemic pigs

Methods 18 male domestic pigs were randomly assigned to 3 groups: one normal control group with a normal diet (Normal, $n=6$ ) and two hypercholesterolemic groups $(\mathrm{HC}, \mathrm{n}=12)$ fed with atherosclerosis-inducing cholesterol-rich chow diet, one of which received EECP (HC, HC+EECP, $\mathrm{n}=6$, respectively). Pigs in the HC+EECP group were treated with EECP for $2 \mathrm{~h}$ every other day for $36 \mathrm{~h}$. In the end of the study, the animals were sacrificed, and the thoracic and abdominal aortas harvested. The thoracic aortas were sampled for both scanning and transmission electronic microscopy (SEM and TEM)whereas the abdominal aortas were stained in Sudan-III of fatty streak for macroscopic evaluation. Vascular endothelial cells (VECs) were isolated from the thoracic aorta by collagenase. TUNEL was used to detect the apoptotic index of VECs. The abdominal aortas were collected for histopathological studies.

Results Fatty streaks or plaques were hardly found in the normal group but clearly observable in the HC group. Atherosclerotic lesions were much less severe in the EECP group than in the HC group. SEM analysis revealed that aortic VECs were irregularly arrayed, markedly desquamated, and shrank into smaller size, which indicated apoptotic events resulting in remarkable damage of endothelium in HC group. In contrast, the VECs in HC+EECP group were arrayed in a relatively streamline fashion, less desquamated and shrank, and manifested comparatively mild endothelial damage. TEM examination of aortas in HC group showed desquamated VECs loosely attached to the matrix along with foam cells, which indicated intimal damage. Apoptotic VECs at early, middle, late stage and even apoptotic bodies were visible on intimal surface. But these changes were relatively mild in EECP-treated animals. The apoptotic index in the $\mathrm{HC}+\mathrm{EECP}$ group was significantly lower than that of the HC group, but still higher than that of the Normal group $((177 \pm 12) \%$, $(237 \pm 23) \%$, $(127 \pm 36) \%$, respectively, $\mathrm{p}<0.05)$.

Conclusions EECP alleviates hypercholesterolaemia-induced atherosclerotic damage to the vascular intima and endothelium, and protects VECs from apoptosis, thereby delaying the progression of early atherosclerotic lesions. The therapeutic benefit of EECP in terms of endothelial protection may be attributed to the inhibition of VEC apoptosis. e0188 ENALAPRIL, IRBESARTAN AND ANG-(1-7) PREVENT ATRIAL TACHYCARDIA-INDUCED SODIUM CHANNEL REMODELLING

doi:10.1136/hrt.2010.208967.188

Wang Xuewen, Li Jian, Xu Zhao, Yang Wansong, Li Guangping. Department of Cardiology, Tianjin Institute of Cardiology, Second Hospital of Tianjin Medical University, Tianjin, China

Purpose Recent studies indicated that the activation of reninangiotensin system (RAS) played an important role in the development and recurrence of atrial fibrillation (AF). Angiotensin II (Ang II) plays a central role in the process of atrial electrical remodelling (AER). Some studies on interfering with RAS have demonstrated positive effects to prevent episodes of AF both in animals and in humans. Angiotension-(1-7) (Ang-(1-7)) is a bioactive component of RAS which can counterbalance most of effects of Ang II. In the present study, the effects of ACEI enalapril, ARB irbesartan and Ang-(1-7) on $\mathrm{Na}^{+}$current $\left(\mathrm{I}_{\mathrm{Na}}\right)$ densities and $\mathrm{Na}^{+}$channel (Nav1.5) mRNA expression were examined in a canine chronic model of AF induced by rapid atrial pacing.

Methods For this study, 30 mongrel dogs of either sex weighing between 11 and $15 \mathrm{~kg}$ were randomly assigned to sham, paced, paced+enalapril, paced+irbesartan and paced + Ang-(1-7) group, six dogs in each. Rapid atrial pacing at $500 \mathrm{bpm}$ was maintained for 2 weeks, while the dogs in sham group underwent pacemaker implantation, but the pacemakers were not activated to provide atrial pacing. During the pacing, the dogs in enalapril, irbesartan or Ang-(1-7) group received enalapril (2 mg/Kg/d), irbesartan (60 mg/ $\mathrm{Kg} / \mathrm{d})$ or Ang-(1-7) $(6 \mu \mathrm{g} / \mathrm{Kg} / \mathrm{h})$, respectively. The whole-cell patchclamp technique was used to record $\mathrm{I}_{\mathrm{Na}}$ and RT-PCR was applied to assess possible underlying changes in cardiac $\mathrm{Na}^{+}$channels.

Results $\mathrm{I}_{\mathrm{Na}}$ densities were reduced by $46.96 \%$ in paced group $(-32.65 \pm 10.92 \mathrm{pA} / \mathrm{pF})$ compared with sham group $(-61.56 \pm 14.17$ $\mathrm{pA} / \mathrm{pF}, \mathrm{p}<0.05)$. The half-activation voltage $\left(\mathrm{V}_{1 / 2 \mathrm{act}}\right)$ and half-inactivation voltage $\left(\mathrm{V}_{1 / 2 \text { inact }}\right)$ of $\mathrm{I}_{\mathrm{Na}}$ were not altered in paced group ( $p>0.05$ vs sham). Enalapril $(-44.11 \pm 16.76 \mathrm{pA} / \mathrm{pF})$, irbesartan $(-65.24 \pm 14.79 \mathrm{pA} / \mathrm{pF})$ and Ang-(1-7) (-66.56 $\pm 18.08 \mathrm{pA} / \mathrm{pF})$ increased $\mathrm{I}_{\mathrm{Na}}$ by $35.10 \%, 99.82 \%$ and $103.86 \%(\mathrm{p}<0.05$ vs paced group), respectively. Enalapril, irbesartan and Ang-(1-7) hyperpolarized $V_{1 / 2 a c t}$ compared with sham and paced group $(p<0.05)$. The difference of $V_{1 / 2 \text { inact }}$ among five groups had no statistical significance. Compared with sham group, Nav1.5 $\alpha$ subunit mRNA abundance decreased dramatically in paced group $(p<0.05)$. Enalapril and irbesartan prevented the decrease of Nav1.5 $\alpha$ subunit mRNA expression compared with paced group $(p<0.05)$. Ang- $(1-7)$ had no effects on the decrease of Nav1.5 $\alpha$ subunit mRNA expression ( $p>0.05$ vs paced group).

Conclusion Enalapril, irbesartan and Ang-(1-7) increase $\mathrm{I}_{\mathrm{Na}}$ densities and contribute to improving intra-atrial conduction and decreasing the likelihood AF maintains. Hence, counterbalance the Ang II actions may represent an important tool to prevent atrial ionic remodelling, and perhaps a novel therapeutic approach to the prevention of $\mathrm{AF}$.

\section{e0189 IN VIVO SPATIOTEMPORAL VISUALISATION AND QUANTIFICATION OF MESENCHYMAL STEM CELLS WITH ROSUVASTATIN IN HINDLIMB ISCHAEMIA MICE BY 3-DIMENSIONAL MOLECULAR IMAGING}

doi:10.1136/hrt.2010.208967.189

${ }^{1}$ Fan Weiwei, ${ }^{1}$ Cao Feng, ${ }^{2}$ Liu Junting, ${ }^{2}$ Ma Xiaopeng, ${ }^{2}$ Li Xiangsi, ${ }^{1}$ Li Shuang, ${ }^{1}$ Zhang Rongqing, ${ }^{1}$ Li Congye, ${ }^{2}$ Tian Jie. ${ }^{1}$ Xijing Hospital; ${ }^{2}$ Xidian University

Background Stem cell therapy has generated much interest in improving the function of ischaemic myocardium and peripheral 